\title{
On differential equations classifying a warped product submanifold of cosymplectic space forms
}

\author{
Akram Ali ${ }^{1}$, Fatemah Mofarreh ${ }^{2 *}$, Rifaqat Ali $^{3}$ and Irfan Anjum Badruddin ${ }^{4}$
}

\section{"Correspondence:}

fyalmofarrah@pnu.edu.sa

${ }^{2}$ Mathematical Science

Department, Faculty of Science, Princess Nourah bint Abdulrahman University, Riyadh, 11546, Saudi Arabia

Full list of author information is available at the end of the article

\begin{abstract}
In the present paper, we extend the study of (Ali et al. in J. Inequal. Appl. 2020:241, 2020) by using differential equations (García-Río et al. in J. Differ. Equ. 194(2):287-299, 2003; Pigola et al. in Math. Z. 268:777-790, 2011; Tanno in J. Math. Soc. Jpn. 30(3):509-531, 1978; Tashiro in Trans. Am. Math. Soc. 117:251-275, 1965), and we find some necessary conditions for the base of warped product submanifolds of cosymplectic space form $\widetilde{M}^{2 m+1}(\epsilon)$ to be isometric to the Euclidean space $\mathbb{R}^{n}$ or a warped product of complete manifold $N$ and Euclidean space $\mathbb{R}$.
\end{abstract}

MSC: $53 C 40$

Keywords: C-totally real warped products; Cosymplectic space forms; Ordinary differential equation; Isometric immersion

\section{Background and motivation}

In [11, 12, 17-19], the authors gave the characterizations of Euclidean spaces by analyzing a differential equation. They have shown that a function $\psi$ which is non-constant on a complete manifold $\left(\Omega^{n}, g\right)$ agrees with the following equation:

$$
\nabla^{2} \psi+c g=0
$$

if and only if $\left(\Omega^{n}, g\right)$ is isometric to the Euclidean spaces $\mathbb{R}^{n}$, where $c$ is any positive constant. There is another characterization by using differential equation which was discovered by Río, Kupeli, and Unal [12]. They demonstrated that the complete Riemannian manifold $\left(\Omega^{n}, g\right)$ is isometric to the warped product of a complete Riemannian manifold $N$ and an Euclidean line $\mathbb{R}$ with warping function $\theta$ accomplishes the differential equation

$$
\frac{d^{2} \theta}{d t^{2}}+\lambda_{1} \theta=0
$$

(c) The Author(s) 2021. This article is licensed under a Creative Commons Attribution 4.0 International License, which permits use, sharing, adaptation, distribution and reproduction in any medium or format, as long as you give appropriate credit to the original author(s) and the source, provide a link to the Creative Commons licence, and indicate if changes were made. The images or other third party material in this article are included in the article's Creative Commons licence, unless indicated otherwise in a credit line to the material. If material is not included in the article's Creative Commons licence and your intended use is not permitted by statutory regulation or exceeds the permitted use, you will need to obtain permission directly from the copyright holder. To view a copy of this licence, visit http://creativecommons.org/licenses/by/4.0/. 
if and only if there exists a real-valued non-constant function $\psi$ associated with the negative eigenvalue $\lambda_{1}<0$ that has the solution of the following differential equation:

$$
\Delta \psi+\lambda_{1} \psi=0
$$

These types of complete space classifications are extremely significant and were researched by several mathematicians (see [2, 6, 9-11, 16]). For example, by using (1.1), Al-Dayel, Deshmukh, and Belova [3] showed that a connected and complete Riemannian manifold $\left(\Omega^{n}, g\right)$ is isometric to $\mathbb{R}^{n}$ if and only if the nontrivial concircular vector field $\mathbf{u}$ along the function $\psi$ satisfies $R(\nabla \psi, \nabla \psi)=0$ or $\Delta \mathbf{u}=\mathbf{0}$. In [7], Chen and Deshmukh proved that a complete Riemannian manifold admits a concurrent vector field if and only if it is isometric to an Euclidean space by using (1.1). Similarly, in [8] it has been shown that $\left(\Omega^{n}, g\right)$ is isometric to the Euclidean space if and only if $\left(\Omega^{n}, g\right)$ permits the nontrivial gradient conformal vector field which is named Jacobi-type vector field. On the other hand, Matsuyama [14] derived a characterization such that the complete totally real submanifold $\Omega^{n}$ of the complex projective space $C \mathbb{P}^{n}$ with bounded Ricci curvature admits a function $\psi$ satisfying (1.3) for $\lambda_{1} \leq n$, then $\Omega^{n}$ is isometric to the hyperbolic space component that is connected if $(\nabla \psi)_{x}=0$ or it is isometric to the warped product of the complete Riemannian manifold and the Euclidean line if $\nabla \psi$ is non-vanishing, where the warping function $\theta$ on $\mathbb{R}$ ensures equation (1.2). Also, similar results have been obtained for generalized Sasakian space forms by Jamali and Shahid [13].

In the present paper, by using the Chen-Ricci inequality which was derived in [4], and influenced by the studies in $[1-3,6,13]$, we derive similar characterization for C-totally real warped product submanifolds of cosymplectic space forms as rigidity theorems.

\section{Notations and formulas}

The almost contact metric manifold $(\widetilde{M}, g)$ with Riemannian metric $g$ satisfies the conditions

$$
\begin{aligned}
& \phi^{2}=-I+\xi \otimes \eta, \\
& \eta(\xi)=1, \quad \phi(\xi)=0, \quad \eta \circ \phi=0, \\
& g\left(\phi W_{1}, \phi W_{2}\right)=g\left(W_{1}, W_{2}\right)-\eta\left(W_{1}\right) \eta\left(W_{2}\right), \\
& \eta\left(W_{1}\right)=g\left(W_{1}, \xi\right),
\end{aligned}
$$

for the almost contact structure $(\phi, \eta, \xi)$ and $\forall W_{1}, W_{2} \in \Gamma(T \widetilde{M})$. The manifold $\widetilde{M}^{2 m+1}$ is defined to be a cosymplectic manifold if the following relation holds:

$$
\left(\widetilde{\nabla}_{W_{1}} \phi\right) W_{2}=0
$$

for every $W_{1}, W_{2} \in \Gamma(T \widetilde{M})$, where $\widetilde{\nabla}$ denotes the Riemannian connection with concern of the metric $g$. A cosymplectic space form is the cosymplectic manifold considering the constant $\phi$-sectional curvature $\epsilon$, it is also defined as $\widetilde{M}^{2 m+1}(\epsilon)$; see [Eq. (6.2) in [5]], where the Riemannian curvature tensor of $\widetilde{M}^{2 m+1}(\epsilon)$ is defined in detail.

In case the structure field $\xi$ is perpendicular to the submanifold $\Omega^{n}$ in $\widetilde{M}^{2 m+1}(\epsilon), \Omega^{n}$ is a C-totally real submanifold of $\widetilde{M}^{2 m+1}(\epsilon)$; also this case $\phi$ maps any tangent space of $\Omega^{n}$ 
into its correspondent to the normal space (see $[3,5])$. Now, we remember the Bochner formula for a differential function on a Riemannian manifold $\Omega^{n}$, that is, $\psi: \Omega^{n} \rightarrow \mathbb{R}$, we have

$$
\frac{1}{2} \Delta|\nabla \psi|^{2}=\operatorname{Ric}_{\Omega^{n}}(\nabla \psi, \nabla \psi)+|\operatorname{Hess}(\psi)|^{2}+g(\nabla(\Delta \psi), \nabla \psi)
$$

such that the Ricci tensor of $\Omega^{n}$ is denoted by Ric. Now, let $\psi$ be a differential function defined on $\Omega$. Thus, the gradient $\nabla f$ is given as follows:

$$
\text { (i) } g(\nabla \psi, X)=X \psi, \quad \text { and } \quad \text { (ii) } \quad \nabla \psi=\sum_{i=1}^{n} e_{i}(\psi) e_{i} \text {. }
$$

The Laplacian $\Delta \psi$ of $\psi$ is also given by

$$
\Delta \psi=\sum_{i=1}^{n}\left\{\left(\nabla_{e_{i}} e_{i}\right) \psi-e_{i}\left(e_{i}(\psi)\right)\right\}=-\sum_{i=1}^{n} g\left(\nabla_{e_{i}} \operatorname{grad} \psi, e_{i}\right)=-\operatorname{traceHess}(\psi) .
$$

Theorem 2.1 (Green's theorem [20]) Let $\Omega$ be a compact oriented Riemannian manifold without boundary, and let $\psi$ be a differential function, then the following formula holds:

$$
\int_{M} \Delta \psi \mathrm{d} V=0
$$

where $\mathrm{d} V$ denotes the volume of $\Omega$.

\section{The main results}

To prove our main result, the next lemma which is proved in [4], is stated.

Lemma 3.1 ([4]) Suppose that $\widetilde{M}^{2 m+1}(\epsilon)$ is a cosymplectic space form, and let $\Phi: \Omega^{n}=\mathbb{B} \times_{f}$ $\mathbb{F} \longrightarrow \widetilde{M}^{2 m+1}(\epsilon)$ be a $\mathbb{B}$-minimal C-totally real isometric immersion of a warped product submanifold $\Omega^{n}$ into $\widetilde{M}^{2 m+1}(\epsilon)$. Then the Ricci inequality is given by

$$
\mathcal{R i c}(X)+q \Delta \ln f \leq \frac{n^{2}}{4}\|H\|^{2}+q\|\nabla \ln f\|^{2}+\frac{\epsilon}{4}\{p q+n-1\}
$$

for every unit vector $X \in T_{x} \Omega^{n}$, where $p=\operatorname{dim} \mathbb{B}$ and $q=\operatorname{dim} \mathbb{F}$. The qualities in the above inequality have been discussed in detail in [4].

The next compositions will be used to the end of this paper: 'CSF' as cosymplectic space form, 'WF' as warping function, and 'WPS' as warped product submanifold. More precisely, we give the next theorem.

Theorem 3.1 Let $\Phi: \Omega^{n}=\mathbb{B} \times_{f} \mathbb{F} \longrightarrow \mathbb{M}^{2 m+1}(\epsilon)$ be a $\mathbb{B}$-minimal C-totally real isometric immersion of the complete WPS $\Omega^{n}$ into the $\operatorname{CSF} \mathbb{M}^{2 m+1}(\epsilon)$ such that Ricci curvature is bounded below by a positive constant $K>0$. Then $\mathbb{B}$ is isometric to an Euclidean space $\mathbb{R}^{p}$ if the following equality holds:

$$
\left(\lambda_{1}+q\right) K=\lambda_{1}\left\{\frac{q \lambda_{1}}{p}+\frac{n^{2}}{4}\|H\|^{2}+\frac{\epsilon}{4}(p q+n-1)\right\},
$$

where $f: \mathbb{B} \longrightarrow \mathbb{R}$ is a real-valued function and called a warping function. 
Proof In particular, $\psi=\ln f$ such that $f: \mathbb{B} \longrightarrow \mathbb{R}$ and Eq. (3.1) gives

$$
\mathcal{R} i c(X)+q \Delta \psi \leq \frac{n^{2}}{4}\|H\|^{2}+q\|\nabla \psi\|^{2}+\frac{\epsilon}{4}\{p q+n-1\}
$$

Assuming that the Ricci curvature is bounded below with any positive constant $K>0$, that is, $\operatorname{Ric}(X) \geq K$, we have

$$
K+q \Delta \psi \leq \frac{n^{2}}{4}\|H\|^{2}+q\|\nabla \psi\|^{2}+\frac{\epsilon}{4}\{p q+n-1\} .
$$

One of the most famous results connecting the curvature and topology of the complete Riemannian manifold $\Omega^{n}$ is the famous theorem of Myers [15], which states that if the Ricci curvature with regard to unit vectors on $\Omega^{n}$ is bounded with a positive constant $K>0$, then $\Omega^{n}$ is compact. This implies that $\Omega^{n}$ is compact, then taking integration (3.3) and using Green's lemma, we find that

$$
\operatorname{Vol}\left(\Omega^{n}\right) K \leq \frac{n^{2}}{4} \int_{\Omega^{n}}|\mathbb{H}|^{2} d V+q \int_{\Omega^{n}}|\nabla \psi|^{2} d V+\int_{\Omega^{n}} \frac{\epsilon}{4}(p q+n-1) d V
$$

This can be written as

$$
\int_{\Omega^{n}}|\nabla \psi|^{2} d V \geq \frac{K}{q} \operatorname{Vol}\left(\Omega^{n}\right)-\frac{n^{2}}{4 q} \int_{\Omega^{n}}|\mathbb{H}|^{2} d V-\frac{1}{q} \int_{\Omega^{n}} \frac{\epsilon}{4}(p q+n-1) d V
$$

On the other hand, we have

$$
|\operatorname{Hess}(\psi)-t I|^{2}=|\operatorname{Hess}(\psi)|^{2}+t^{2}|I|^{2}-2 \operatorname{tg}(I, \operatorname{Hess}(\psi)),
$$

which leads to the following: $g\left(\operatorname{Hess}(\psi), I^{*}\right)=\operatorname{tr}\left(I^{*} \operatorname{Hess}(\psi)\right)=\operatorname{trHess}(\psi)=-\Delta \psi$ and $(2.3)$

$$
|\operatorname{Hess}(\psi)-t I|^{2}=2 t \Delta \psi+t^{2} p+|\operatorname{Hess}(\psi)|^{2}
$$

Substituting $t=\frac{\lambda_{1}}{p}$ and integrating the preceding equation with respect to the volume element $d V$, we obtain

$$
\int_{\Omega^{n}}\left|\operatorname{Hess}(\psi)-\frac{\lambda_{1}}{p} I\right|^{2} d V=\int_{\Omega^{n}}|\operatorname{Hess}(\psi)|^{2} d V+\int_{\Omega^{n}} \frac{\lambda_{1}^{2}}{p} d V
$$

Applying the integration in Bochner formula (2.1) with the fact that $\Delta \psi=\lambda_{1} \psi$, we have

$$
\int_{\Omega^{n}}|\operatorname{Hess}(\psi)|^{2} d V=-\lambda_{1} \int_{\Omega^{n}} \mid \nabla \psi \|^{2} d V-\int_{\Omega^{n}} \operatorname{Ric}(\nabla \psi, \nabla \psi) d V .
$$

Combining Eqs. (3.5) and (3.6), we derive

$$
\int_{\Omega^{n}}\left|\operatorname{Hess}(\psi)-\frac{\lambda_{1}}{p} I\right|^{2} d V=\int_{\Omega^{n}} \frac{\lambda_{1}^{2}}{p} d V-\lambda_{1} \int_{\Omega^{n}} \mid \nabla \psi \|^{2} d V-\int_{\Omega^{n}} \operatorname{Ric}(\nabla \psi, \nabla \psi) d V
$$


As we assumed that $\operatorname{Ric}(\nabla \psi, \nabla \psi) \geq K$ for $K>0$, then we proceed to the next expression

$$
\int_{\Omega^{n}}\left|\operatorname{Hess}(\psi)-\frac{\lambda_{1}}{p} I\right|^{2} d V \leq \int_{\Omega^{n}} \frac{\lambda_{1}^{2}}{p} d V-\lambda_{1} \int_{\Omega^{n}} \mid \nabla \psi \|^{2} d V-K \operatorname{Vol}\left(\Omega^{n}\right) .
$$

Inserting Eq. (3.4) into the above equation, we derive

$$
\begin{aligned}
\int_{\Omega^{n}}\left|\operatorname{Hess}(\psi)-\frac{\lambda_{1}}{p} I\right|^{2} d V \leq & \int_{\Omega^{n}} \frac{\lambda_{1}^{2}}{p} d V-\int_{\Omega^{n}}\left(\frac{\lambda_{1} K}{q}+K\right) d V \\
& +\frac{\lambda_{1} n^{2}}{4 q} \int_{\Omega^{n}}|\mathbb{H}|^{2} d V+\frac{\lambda_{1}}{q} \int_{\Omega^{n}} \frac{\epsilon}{4}(p q+n-1) d V .
\end{aligned}
$$

If (3.2) is satisfied, then (3.7) implies that

$$
\left|\operatorname{Hess}(\psi)-\frac{\lambda_{1}}{p} I\right|^{2}=0
$$

Hence, we get

$$
\operatorname{Hess}(\psi)(V, V)=c g(V, V)
$$

for any $V \in \Gamma(\mathbb{B})$ with constant $c=\frac{\lambda_{1}}{p}$. Therefore, by implementing Tashiro theorems [17, $19]$, we analyze that $\mathbb{B}$ is isometric to an Euclidean space $\mathbb{R}^{p}$.

The next result comes from the motivation by the study of Río, Kupeli, and Unal [12]. We prove the following.

Theorem 3.2 Let $\Phi: \Omega^{n}=\mathbb{B} \times_{f} \mathbb{F} \rightarrow \mathbb{M}^{2 m+1}(\epsilon)$ be a $\mathbb{B}$-minimal C-totally real isometric immersion of a complete WPS $\Omega^{n}$ into the CSF $\mathbb{M}^{2 m+1}(\epsilon)$ such that the Ricci curvature is bounded below by a positive constant $K>0$ and satisfies the following assumption:

$$
\left|\nabla^{2} \psi\right|^{2}=\frac{\lambda_{1}}{4 p q}\left\{\epsilon(1-p q-n)+4 K-n^{2}|\mathbb{H}|^{2}\right\}
$$

for $\lambda_{1}<0$. Then $\mathbb{B}$ is isometric to the warped product of the form $\mathbb{R} \times{ }_{\theta} N$ with the warping function $\theta$ satisfying the following differential equation:

$$
\frac{d^{2} \theta}{d t^{2}}+\lambda_{1} \theta=0
$$

Proof Let us define the following equation with $\psi=\ln f$, we have

$$
|t \psi I+\operatorname{Hess}(\psi)|^{2}=t^{2}(\psi)^{2}|I|^{2}+|\operatorname{Hess}(\psi)|^{2}+2 t \psi g(I, \operatorname{Hess}(\psi)) .
$$

However, it is known that $|I|^{2}=\operatorname{trace}\left(\mathrm{II}^{*}\right)=p$, also $g\left(\operatorname{Hess}(\psi), I^{*}\right)=\operatorname{tr}\left(I^{*} \operatorname{Hess}(\psi)\right)=$ $\operatorname{trHess}(\psi)=-\Delta \psi$ from (2.3). Then the preceding equation takes the form

$$
|t \psi I+\operatorname{Hess}(\psi)|^{2}=|\operatorname{Hess}(\psi)|^{2}+p t^{2}(\psi)^{2}-2 t \psi \Delta \psi
$$


If $\psi$ is an eigenfunction associated with the eigenvalue $\lambda_{1}$ such that $\Delta \psi=\lambda_{1} \psi$ with implemented integration, then we get the following from the preceding equation:

$$
\int_{\Omega^{n}}|t \psi I+\operatorname{Hess}(\psi)|^{2} d V=\int_{\Omega^{n}}|\operatorname{Hess}(\psi)|^{2} d V+\int_{\Omega^{n}}\left(p t^{2}-2 t \lambda_{1}\right)(\psi)^{2} d V .
$$

On the other hand, we obtain

$$
\Delta \frac{\psi^{2}}{2}=\psi \Delta \psi-|\nabla \psi|^{2} .
$$

Applying Green's theorem along with $\Delta \psi=\lambda_{1} \psi$, we arrive at

$$
\int_{\Omega^{n}}(\psi)^{2} d V=\frac{1}{\lambda_{1}} \int_{\Omega^{n}}|\nabla \psi|^{2} d V
$$

From (3.9) and (3.10), we find that

$$
\int_{\Omega^{n}}|\operatorname{Hess}(\psi)+t \psi I|^{2} d V=\int_{\Omega^{n}}|\operatorname{Hess}(\psi)|^{2} d V+\int_{\Omega^{n}}\left(\frac{p t^{2}}{\lambda_{1}}-2 t\right)|\nabla \psi|^{2} d V .
$$

In particular, $t=\frac{\lambda_{1}}{p}$ in (3.11), and taking integration, we get

$$
\int_{\Omega^{n}}\left|\operatorname{Hess}(\psi)+\frac{\lambda_{1}}{p} \psi I\right|^{2} d V=\int_{\Omega^{n}}|\operatorname{Hess}(\psi)|^{2} d V-\frac{\lambda_{1}}{p} \int_{\Omega^{n}}|\nabla \psi|^{2} d V .
$$

Again taking integration on (3.1) and involving Green's theorem, we have

$$
\int_{\Omega^{n}} \mathcal{R} i c_{M}(X) d V \leq \frac{n^{2}}{4} \int_{\Omega^{n}}|\mathbb{H}|^{2} d V+q \int_{\Omega^{n}}|\nabla \psi|^{2} d V+\int_{\Omega^{n}} \frac{\epsilon}{4}(p q+n-1) d V .
$$

From (3.12) and (3.13), we derive

$$
\begin{aligned}
\frac{1}{q} \int_{\Omega^{n}} \operatorname{Ric}_{M}(X) d V \leq & \frac{n^{2}}{4 q} \int_{\Omega^{n}}|\mathbb{H}|^{2} d V-\frac{p}{\lambda_{1}} \int_{\Omega^{n}}\left|\operatorname{Hess}(\psi)+\frac{\lambda_{1}}{n} \psi I\right|^{2} d V \\
& +\frac{p}{\lambda_{1}} \int_{\Omega^{n}}|\operatorname{Hess}(\psi)|^{2} d V+\int_{\Omega^{n}} \frac{\epsilon}{4}\left(p+1+\frac{p-1}{q}\right) d V .
\end{aligned}
$$

As we considered that the Ricci curvature is bounded $\operatorname{Ric}(X) \geq K$ as $K>0$, thus the preceding equation implies

$$
\begin{aligned}
\int_{\Omega^{n}}\left|\operatorname{Hess}(\psi)+\frac{\lambda_{1}}{p} \psi I\right|^{2} d V \leq & \frac{n^{2} \lambda_{1}}{4 p q} \int_{\Omega^{n}}|\mathbb{H}|^{2} d V+\int_{\Omega^{n}}|\operatorname{Hess}(\psi)|^{2} d V-\frac{\lambda_{1}}{p q} \int_{\Omega^{n}} K d V \\
& +\frac{\lambda_{1}}{p} \int_{\Omega^{n}} \frac{\epsilon}{4}\left(p+1+\frac{p-1}{q}\right) d V
\end{aligned}
$$

it is equivalent to the following:

$$
\begin{aligned}
\int_{\Omega^{n}}\left|\operatorname{Hess}(\psi)+\frac{\lambda_{1}}{p} \psi I\right|^{2} d V \leq & \int_{\Omega^{n}}|\operatorname{Hess}(\psi)|^{2} d V \\
& +\frac{\lambda_{1}}{p} \int_{\Omega^{n}}\left\{\frac{n^{2}}{4 q}|\mathbb{H}|^{2}+\frac{\epsilon}{4}\left(p+1+\frac{p-1}{q}\right)-\frac{K}{q}\right\} d V .
\end{aligned}
$$


The above equation implies that

$$
\begin{aligned}
& \int_{\Omega^{n}}\left|\operatorname{Hess}(\psi)+\frac{\lambda_{1}}{p} \psi I\right|^{2} d V \\
& \quad \leq \int_{\Omega^{n}}\left\{\frac{\lambda_{1} n^{2}}{4 p q}|\mathbb{H}|^{2}+\frac{\lambda_{1} \epsilon}{4 p}\left(p+1+\frac{p-1}{q}\right)-\frac{\lambda_{1} K}{p q}+|\operatorname{Hess}(\psi)|^{2}\right\} d V .
\end{aligned}
$$

Our assumption (3.8) is satisfied, that is,

$$
\lambda_{1} n^{2}|\mathbb{H}|^{2}+4 p q|\operatorname{Hess}(\psi)|^{2}=\lambda_{1}(\epsilon(1-p q-n)+4 K)
$$

From (3.14) and (3.15), we get the following:

$$
\left|\operatorname{Hess}(\psi)+\frac{\lambda_{1}}{p} \psi I\right|^{2} \leq 0
$$

The above equation gives us

$$
\operatorname{Hess}(\psi)+\frac{\lambda_{1}}{p} \psi I=0
$$

Tracing the preceding equation, we derive

$$
\Delta \psi+\lambda_{1} \psi=0
$$

According to [12], the base $\mathbb{B}$ is isometric to the connected components of hyperbolic space if $(\nabla \psi)_{x}=0$. But $(\nabla \psi)_{x}=0$ leads to a contradiction as $\Omega^{n}$ is a nontrivial warped product. Hence $\mathbb{B}$ is isometric to the warped product of the type $\mathbb{R} \times_{\theta} N$, where $N$ is the complete Riemannian manifold, also $\mathbb{R}$ is an Euclidean line. Moreover, the warping function $\theta$ ensures the following differential equation:

$$
\frac{d^{2} \theta}{d t^{2}}+\lambda_{1} \theta=0
$$

This proof is completed.

For the minimality case, that is, $\|H\|^{2}=0$, we give the following corollary.

Corollary 3.1 Let $\Psi: \Omega^{n}=\mathbb{B} \times_{f} \mathbb{F} \rightarrow \mathbb{M}^{2 m+1}(\epsilon)$ be a $C$-totally real minimal isometric immersion of a complete WPS $\Omega^{n}$ into the CSF $\mathbb{M}^{2 m+1}(\epsilon)$ such that the Ricci curvature is bounded below of a positive constant $K>0$ which satisfies the condition

$$
\left(\lambda_{1}+q\right) K=\lambda_{1}\left\{\frac{q \lambda_{1}}{p}+\frac{\epsilon}{4}(p q+n-1)\right\} .
$$

Then $\mathbb{B}$ is isometric to an Euclidean space $\mathbb{R}^{p}$.

We give the following corollary by using Theorem 3.2. 
Corollary 3.2 Let $\Phi: \Omega^{n}=\mathbb{B} \times_{f} \mathbb{F} \longrightarrow \mathbb{M}^{2 m+1}(\epsilon)$ be a C-totally real minimal isometric immersion of a complete WPS $\Omega^{n}$ into the CSF $\mathbb{M}^{2 m+1}(\epsilon)$ such that Ricci curvature is bounded below of a positive constant $K>0$ satisfying the assumption

$$
\left|\nabla^{2} \psi\right|^{2}=\frac{\lambda_{1}}{4 p q}\{\epsilon(1-p q-n)+4 K\} .
$$

Then $\mathbb{B}$ is isometric to the warped product of the form $\mathbb{R} \times_{\theta} N$ with the warping function $\theta$ satisfying differential equation (1.2).

Classifying Dirichlet energy of smooth functions is treated as an integral part in the field of physics and engineering. Moreover, Dirichlet energy is formulated to be an equivalent of kinetic energy. Let $\psi$ be any real-valued smooth function on a compact manifold, then the Dirichlet energy is defined by

$$
\mathcal{E}(\psi)=\frac{1}{2} \int\|\nabla \psi\|^{2} d V
$$

By using the above formula and Lemma 3.1, we give the following theorem.

Theorem 3.3 Suppose that $\widetilde{M}^{2 m+1}(\epsilon)$ is a cosymplectic space form, and let $\Phi: \Omega^{n}=\mathbb{B} \times_{f}$ $\mathbb{F} \longrightarrow \widetilde{M}^{2 m+1}(\epsilon)$ be a C-totally real immersion of the warped product submanifold $\Omega^{n}$ into $\widetilde{M}^{2 m+1}(\epsilon)$ such that the base $\mathbb{B}$ is minimal. Then the Dirichlet energy inequality is given by

$$
\int_{\Omega^{n}} \operatorname{Ric}(X) d V \leq \frac{n^{2}}{4} \int_{\Omega^{n}}\|H\|^{2} d V+2 q \mathcal{E}(\psi)+\{p q+n-1\} \int_{\Omega^{n}} \frac{\epsilon+3}{4} d V
$$

for every unit vector $X \in T_{x} \Omega^{n}$, where $p=\operatorname{dim} \mathbb{B}$ and $q=\operatorname{dim} \mathbb{F}$.

Proof Taking integration in Eq. (3.1) and using Green's lemma with (3.17), we get the required result (3.18). This completes the proof of the theorem.

\section{Acknowledgements}

The first and third authors would like to express their gratitude to Deanship of Scientific Research at King Khalid University, Saudi Arabia for providing for funding this work through the General Research Project under grant number (G.R.P-316-42). This research was funded by the Deanship of Scientific Research at Princess Nourah bint Abdulrahman University through the Fast-track Research Funding Program to support publication in the top journal (Grant no. 42-FTTJ-03).

\section{Funding}

The first and third authors would like to express their gratitude to Deanship of Scientific Research at King Khalid University, Saudi Arabia for providing for funding this work through the General Research Project under grant number (G.R.P-316-42). This research was funded by the Deanship of Scientific Research at Princess Nourah bint Abdulrahman University through the Fast-track Research Funding Program to support publication in the top journal (Grant no. 42-FTTJ-03).

\section{Availability of data and materials}

There is no data use for this manuscript.

\section{Competing interests}

The authors declare that they have no competing interests.

\section{Authors' contributions}

All authors have equal contributions and finalized the paper. All authors read and approved the final manuscript.

\section{Author details}

${ }^{1}$ Department of Mathematics, College of Science, King Khalid University, 9004 Abha, Saudi Arabia. ${ }^{2}$ Mathematical Science Department, Faculty of Science, Princess Nourah bint Abdulrahman University, Riyadh, 11546, Saudi Arabia. ${ }^{3}$ Department of Mathematics, College of Science and Arts, Muhayi, King Khalid University, 61413 Abha, Saudi Arabia.

${ }^{4}$ Department of Mechanical Engineering, College of Engineering, King Khalid University, 9004 Abha, Saudi Arabia. 


\section{Publisher's Note}

Springer Nature remains neutral with regard to jurisdictional claims in published maps and institutional affiliations.

Received: 21 August 2020 Accepted: 19 April 2021 Published online: 29 April 2021

\section{References}

1. Al-Dayel, I., Deshmukh, S., Belova, O.: A remarkable property of concircular vector fields on a Riemannian manifold. Mathematics 8, 469 (2020)

2. Ali, A., Laurian-loan, P., Alkhaldi, A.H.: Ricci curvature on warped product submanifolds in spheres with geometric applications. J. Geom. Phys. 146, 103510 (2019)

3. Ali, A., Laurian-loan, P., Alkhaldi, A.H., Alqahtani, L.S.: Ricci curvature on warped product submanifolds of complex space forms and its applications. Int. J. Geom. Methods Mod. Phys. 16(9), 1950142 (2019)

4. Ali, A., Mofarreh, F., Mior Othman, W.A., Patra, D.S.: Applications of differential equations to characterize the base of warped product submanifolds of cosymplectic space forms. J. Inequal. Appl. 2020, 241 (2020)

5. Ali, A., Ozel, C.: Geometry of warped product pointwise semi-slant submanifolds of cosymplectic manifolds and its applications. Int. J. Geom. Methods Mod. Phys. 14, no. 3, 1750042, 29 (2017)

6. Ali, R., Mofarreh, F., Alluhaibi, N., Ali, A., Ahmad, I.: On differential equations characterizing Legendrian submanifolds of Sasakian space forms. Mathematics 8, 150 (2020)

7. Chen, B.Y., Deshmukh, S.: Some results about concircular vector fields on Riemannian manifolds. Filomat 34(3), 835-842 (2020)

8. Chen, B.Y., Deshmukh, S., Ishan, A.A.: On Jacobi-type vector fields on Riemannian manifolds. Mathematics 7(12), 1139 (2019)

9. Deshmukh, S.: Characterizing spheres and Euclidean spaces by conformal vector fields. Ann. Mat. Pura Appl. 196(6), 2135-2145 (2017)

10. Deshmukh, S., Al-Solamy, F.R.: Conformal gradient vector fields on a compact Riemannian manifold. Colloq. Math. 112(1), 157-161 (2008)

11. Erkekoğlu, F., García-Río, E., Kupeli, D.N., Ünal, B.: Characterizing specific Riemannian manifolds by differential equations. Acta Appl. Math. 76(2), 195-219 (2003)

12. García-Río, E., Kupeli, D.N., Unal, B.: On differential equations characterizing Euclidean sphere. J. Differ. Equ. 194(2), 287-299 (2003)

13. Jamali, M., Shahid, M.H.: Application of Bochner formula to generalized Sasakian space forms. Afr. Math. 29(7-8), 1135-1139 (2018)

14. Matsuyama, Y:: Complete totally real submanifolds of a complex projective space. Differ. Geom.-Dyn. Syst. 20 119-125 (2018)

15. Myers, S.B.: Riemannian manifolds with positive mean curvature. Duke Math. J. 8(2), $401-404$ (1941)

16. Obata, M.: Certain conditions for a Riemannian manifold to be isometric with a sphere. J. Math. Soc. Jpn. 14, 333-340 (1962)

17. Pigola, S., Rimoldi, M., Setti, A.G.: Remarks on non-compact gradient Ricci solitons. Math. Z. 268, 777-790 (2011)

18. Tanno, S.: Some differential equations on Riemannian manifolds. J. Math. Soc. Jpn. 30(3), 509-531 (1978)

19. Tashiro, Y.: Complete Riemannian manifolds and some vector fields. Trans. Am. Math. Soc. 117, 251-275 (1965)

20. Yano, K., Kon, M.: Structures on Manifolds. World Scientific, Singapore (1984)

\section{Submit your manuscript to a SpringerOpen ${ }^{\circ}$ journal and benefit from:}

- Convenient online submission

- Rigorous peer review

- Open access: articles freely available online

- High visibility within the field

- Retaining the copyright to your article

Submit your next manuscript at $\gg$ springeropen.com 